\title{
Prevalência e fatores associados aos transtornos mentais comuns em residentes médicos e da área multiprofissional
}

\author{
Prevalence and factors associated with commom mental \\ disorders in medical and multiprofessional health residents
}

Carla Novaes Carvalho', Djalma Agripino de Melo-Filho², João Alberto Gomes de Carvalho', Ana Carla Guedes de Amorim ${ }^{3}$

\section{RESUMO}

Objetivo: Determinar a prevalência de transtornos mentais comuns (TMC) e sua associação a fatores sociodemográficos e profissionais em residentes de medicina, enfermagem, nutrição e saúde coletiva da cidade do Recife (PE). Métodos: Estudo transversal foi conduzido, em 2007, envolvendo uma amostra aleatória de 178 residentes que responderam a questões sociodemográficas e sobre a formação profissional e ao Self-Reporting Questionnaire (SRQ-20). Calcularam-se as prevalências de TMC e estimaram-se as razões de prevalência (RP) e os intervalos de confiança. Resultados: A prevalência total dos TMC foi de 51,1\% e não se observou associação aos fatores sociodemográficos. A prevalência do evento foi $39 \%$ maior nos médicos que nos não médicos $(p=0,049)$ e $46 \%$ maior em residentes médicos das especialidades cirúrgicas que entre os de enfermagem, nutrição e saúde coletiva $(p=0,048)$. Cinco das queixas do SRQ-20 foram mais frequentes no sexo feminino ( $p<$

\section{Palavras-chave}

Transtornos mentais, saúde mental, residência médica, educação médica.

0,05). Conclusão: Os dados demonstram a elevada magnitude dos TMC nessa população, principalmente nos residentes médicos, e servem para educadores e gestores de serviços de saúde no sentido de viabilizar estratégias para prevenir e recuperar a qualidade de vida dos residentes.

\section{ABSTRACT}

Objective: To determine the prevalence of common mental disorders (CMD) and its association with sociodemographics and professionals resident in medicine, nursing, nutrition and public health from the city of Recife (PE). Methods: A survey was conducted in 2007, involving a random sample of 178 residents, who responded to question about sociodemographics and professional training and the Self-Reporting Questionnaire (SRQ-20). The prevalence of CMD were calculated, also prevalence ratios (PR) and confidence intervals were estimated. Results: The overall prevalence of CMD was $51.1 \%$, and there was no association with sociodemographics factors. The prevalence of the event was 39\% higher among the

1 Universidade Federal de Pernambuco (UFPE), Centro de Ciências da Saúde, Programa de Pós-graduação em Neuropsiquiatria e Ciências do Comportamento. 


\section{Keywords}

Mental disorders, mental

health, internship and

residency, medical

education. physicians than in the non-medical $(p=0,049)$ and $46 \%$ greater in the surgical specialties medical residents than among nursing, nutrition and public health $(p=0,048)$. Five of the SRQ-20 items were more frequent in female $(p<0,05)$. Conclusion: These findings indicate the high magnitude of CMD in this population, especially medical residents and serve to educators and managers of health services in order to define strategies to prevent and recover the mental health and the quality of life of the residents.

\section{INTRODUÇÃO}

O conceito de transtornos mentais comuns (TMC) desenvolveu-se, na década de 1970, por meio de pesquisas sobre adoecimento mental no âmbito da atenção primária em saúde, contexto em que se verifica maior prevalência de transtornos não psicóticos em contraste com o perfil de casos psiquiátricos mais graves do ambiente hospitalar'. A expressão TMC foi criada por Goldberg e Huxley e se refere a sintomas como insônia, fadiga, irritabilidade, esquecimento, dificuldade de concentração e queixas somáticas. Esses autores destacam que os transtornos, apesar de inicialmente não serem graves, causam enorme sofrimento e, posteriormente, podem estar associados à incapacidade e ao absenteísmo no trabalho.

Os TMC, embora não configurem uma entidade clínica específica, descrita em manuais nosológicos, estão relacionados, segundo Mari e Jorge ${ }^{2}$, aos transtornos de ansiedade, somatização e depressão sem sintomas psicóticos, constituindo, portanto, uma dimensão desses fenômenos psicopatológicos, expressa no nível coletivo, cuja abordagem é realizada principalmente pelos estudos epidemiológicos.

Segundo a Organização Mundial de Saúde (OMS) ${ }^{3}$, os transtornos depressivos ocupam o quarto lugar entre as dez primeiras causas de incapacidade no mundo. Eles representam, como categoria diagnóstica única, a principal causa de inaptidão quando se consideram os anos vividos com esse problema.

Os estudos iniciais sobre TMC em países industrializados referiam uma variação na prevalência entre $7 \%$ e 30\%'. No Nordeste do Brasil, investigação realizada, em 1993, em Olinda (PE), encontrou prevalência de 35\% e associação desses transtornos às condições de vida e estrutura ocupacional ${ }^{4}$. Inquérito desenvolvido, em 1994, em Pelotas (RS), revelou que 22,7\% da população adulta da zona urbana sofria de TMC ${ }^{5}$.

Os transtornos mentais são resultado de múltiplos determinantes, destacando-se os de origem genética, comportamentais e ambientais, e se associam a alguns fatores como sexo, faixa etária, situação conjugal, condições de vida e trabalho',4.

O interesse em perscrutar o construto TMC tem crescido para além de sua detecção na população geral. Estudos foram desenvolvidos para mensurar sua magnitude em várias profissões ${ }^{6-8}$, incluindo os profissionais de saúde ${ }^{9-12}$. Pitta ${ }^{9}$ observou prevalência elevada em trabalhadores do setor de hemodiálise $(45,5 \%)$ e da UTI pediátrica $(32,1 \%)$ em um hospital geral de São Paulo. Constata-se uma concordância entre os estudos quando revelam que médicos, enfermeiros, auxiliares de enfermagem e agentes comunitários de saúde constituem grupos expostos à insalubridade mental|9-12.

Segundo Smith et al.13, a magnitude dos transtornos mentais em residentes médicos parece estar aumentando, caracterizando o grupo como de maior risco de adoecimento ${ }^{14,15}$. A residência constitui uma das fases mais estressantes na formação do médico, principalmente durante o primeiro ano de treinamento, quando expõe o jovem treinando a sofrimentos que podem provocar alterações comportamentais indesejáveis ${ }^{15-17}$. Small ${ }^{18}$ descreveu, em residentes médicos, um quadro sindrômico, denominado de house officer stress syndrome, caracterizado por distúrbios cognitivos episódicos, raiva crônica, ceticismo, discórdia familiar, depressão, ideação suicida e suicídio e abuso de drogas.

A residência se caracteriza pelo treinamento em serviço sob supervisão, cuja finalidade é a criação de competência profissional para desenvolver conhecimentos, atitudes e habilidades. O residente exercita a prática médica amparando-se nos saberes científico e tecnológico, ao mesmo tempo em que está em contato permanente com a dor, o sofrimento, a doença e a morte ${ }^{16}$. Nesse processo, configurado pela dualidade trabalhador-aprendiz, o residente enfrenta uma tensão interna constante que tanto pode ajudar no seu aperfeiçoamento quanto atuar como fator desencadeante de transtornos mentais. Antes restrita aos médicos, a modalidade de treinamento se expandiu para enfermeiros, nutricionistas e outras categorias profissionais da área de saúde.

As investigações sobre saúde mental em grupos de profissionais sob treinamento em regime de residência são escassas, ao contrário daquelas desenvolvidas em graduandos ${ }^{19-21}$ ou em grupos profissionalizados ${ }^{12,22}$.

Nesse sentido, esta investigação teve como objetivo determinar a prevalência de transtornos mentais comuns e sua associação com variáveis sociodemográficas e relativas à formação profissional em pós-graduandos dos programas de residência de medicina, enfermagem, nutrição e saúde coletiva da cidade do Recife.

\section{MÉTODO}

\section{Universo e amostra}

O universo do estudo foi formado pelo total de pósgraduandos, do primeiro e segundo anos, matriculados, 
em 2007, nos programas de residência da cidade do Recife (PE), com acesso direto para as áreas de medicina, enfermagem, nutrição e saúde coletiva, gerenciados pela Universidade Federal de Pernambuco (UFPE), Universidade de Pernambuco (UPE), Centro de Pesquisas Aggeu Magalhães (Fiocruz-PE) e Secretaria Estadual de Saúde (SES-PE). Na época de investigação, havia 617 pessoas nessa condição. Para o cálculo amostral, utilizaram-se os seguintes parâmetros: intervalo de confiança de 95\%, erro de amostragem de 10\% e uma prevalência estimada do evento de 50\%, com a finalidade de maximizar o tamanho amostral. O cálculo foi desenvolvido no programa Epi-Info, versão 6,0, obtendo-se um $n=167$. Considerando possíveis perdas, o tamanho da amostra foi aumentado em 20\%, obtendo-se um total de 200 participantes. A composição da amostra foi estratificada segundo a proporção de residentes em cada programa, considerando as diferentes especialidades, medicina, enfermagem, nutrição, saúde coletiva, e ano de treinamento. O procedimento de seleção dos participantes foi aleatório e realizado de modo sistemático a partir dos intervalos de amostragem. Nos casos em que o residente escolhido encontrava-se em férias, em viagem, em algum treinamento fora da cidade ou, ainda, em gozo de licença-maternidade, selecionava-se, na listagem, o nome localizado logo abaixo do seu. Não houve reposição amostral, e quando, após três tentativas, o residente não era encontrado, considerava-se perda.

\section{Desenho de estudo e coleta de dados}

Conduzido entre outubro e dezembro de 2007, o estudo transversal avaliou 178 residentes para detecção de TMC por meio da versão brasileira do Self-Reporting Questionnaire (SRQ-20). Além disso, foram respondidas questões sobre dados sociodemográficos e formação profissional, incluindo idade, sexo, estado conjugal, profissão, ano de conclusão do curso de graduação, nome do programa de residência, ano de treinamento, cidade em que residia antes do treinamento, número de filhos e situação de moradia, que fizeram parte do questionário original procedente da dissertação de mestrado da autora ${ }^{23}$. O instrumento de coleta foi testado em estudo piloto. A coleta dos dados ocorreu no próprio local de trabalho dos participantes, em geral após as reuniões das equipes da residência ou, por meio de agendamento, quando os residentes sorteados não estivessem presentes. Após serem prestadas as orientações sobre a pesquisa e assinado o Termo de Consentimento Livre e Esclarecido, todos os residentes sorteados preenchiam o questionário, em uma sala isolada, sob supervisão direta da autora ou de um dos auxiliares da pesquisa, que permaneciam no local para prestar esclarecimentos. Os questionários foram distribuídos dentro de envelopes padronizados e, após devolução, cada residente os depositava em uma urna, de modo a garantir a confidencialidade do processo.
O SRQ-20 foi desenvolvido por Harding et al. ${ }^{24}$. Quando um instrumento é aplicado, dois aspectos devem ser considerados em sua avaliação: a validade e a confiabilidade. O primeiro, também chamado de acurácia, se refere à sua capacidade de medir o que se propõe medir, e o segundo, também chamado de precisão ou fidedignidade, revela o grau de concordância entre as múltiplas medidas de um mesmo fenômeno. Embora a confiabilidade seja uma condição necessária, ela não é suficiente para avaliar a validade.

Do ponto de vista operacional, a validade do SRQ-20 é aferida tomando-se como padrão-ouro a entrevista psiquiátrica. Nesse sentido, percebe-se variação entre 62,9\% e 90\% de sua sensibilidade e entre $44 \%$ e $95,2 \%$ de sua especificida$\mathrm{de}^{25}$. Em relação à confiabilidade desse instrumento, Santos et al. ${ }^{26}$, usando a fórmula de Kuder-Richardson (KD-20) para avaliar a consistência interna do instrumento, encontraram um coeficiente geral e padronizado de 0,80, semelhante ao do estudo de lacoponi e Mari27.

O SRQ-20 é composto por 20 questões do tipo "sim/não", das quais quatro se referem a queixas somáticas e 16, a sintomas psíquicos. Cada um dos 20 itens pode apresentar como escore 0 ou 1, indicando, respectivamente, ausência ou presença do sintoma nos últimos 30 dias. O ponto de corte uti-

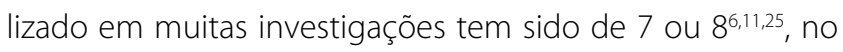
entanto muitos autores defendem o uso de ponto de corte diferenciado entre os sexos, 7 ou 8 para o feminino e 5 ou 6 para o masculino, a fim aumentar a validade, diminuindo os bias de classificação $0^{19,21,24,25,28}$. Neste estudo, considerou-se caso de TMC o participante do sexo masculino que respondeu de modo afirmativo a, no mínimo, seis questões do SRQ-20 ou do sexo feminino que respondeu, dessa maneira, a pelo menos oito questões.

Numa primeira abordagem, os programas de residência foram categorizados segundo áreas: medicina, enfermagem, nutrição e saúde coletiva. Em relação aos programas médicos, buscou-se, ainda, agregá-los segundo a natureza predominante da prática: clínica ou cirúrgica. Assim, as especialidades cujos profissionais desenvolviam rotineiramente a prática cirúrgica, incluindo o ato anestésico, foram classificadas como "cirúrgicas" e as outras foram consideradas "Clínicas". Nesse sentido, procurou-se vincular os profissionais a seus ambientes de trabalho e a inserção no bloco cirúrgico foi um critério demarcador de "exposição". Desse modo, elaborou-se a seguinte classificação: "especialidades cirúrgicas": cirurgia geral, obstetrícia-ginecologia, anestesiologia, oftalmologia, traumatologia-ortopedia e otorrinolaringologia; e "especialidades clínicas": clínica médica, pediatria, radiologia, infectologia, neurologia, psiquiatria e dermatologia.

\section{Plano de descrição e análise}

Os dados foram processados e analisados no programa Epi-Info, versão 6,0 (2001), determinando-se as prevalências de 
TMC, global e específicas, e sua associação com as variáveis sociodemográficas e relativas à formação profissional pela razão de prevalência (RP), cuja significância estatística foi avaliada pelo teste $X^{2}$ ou, quando necessário, pelo teste exato de Fisher, considerando os intervalos de confiança a 95\% e valores do p. A probabilidade máxima de erro para rejeição da hipótese nula foi de $5 \%$.

Todo o processo da pesquisa obedeceu aos princípios éticos dispostos na Resolução no 196/96, do Conselho Nacional de Saúde, do Ministério da Saúde, garantindo aos participantes, entre outros direitos, o seu consentimento livre e esclarecido, sigilo das informações e privacidade. O projeto foi aprovado pelo Comitê de Ética em Pesquisa do Centro de Ciências da Saúde da Universidade Federal de Pernambuco, registro n’287/07, em 16 de outubro de 2007.

\section{RESULTADOS}

Registrou-se uma perda de 11\% (22/200), sete recusaram-se a participar e 15 não foram localizados. O grau de preenchimento médio das questões foi de 99,1\%. A idade média dos participantes foi de $27 \pm 2,43$ anos e $68,5 \%$ eram do sexo feminino. Cerca de $67 \%$ residiam no Recife antes do treina- mento e $88 \%$ moravam com outra(s) pessoa(s). Aproximadamente $74 \%$ eram solteiros e $90 \%$ não tinham filhos. A prevalência global de TMC foi de $51,1 \%$ e não se observou associação estatisticamente significante $(p>0,05)$ com sexo, idade, cidade onde residia antes do treinamento, situação de moradia, estado conjugal e número de filhos (Tabela 1).

A maioria dos residentes era formada de médicos (68\%). Aproximadamente $67 \%$ dos participantes concluíram a graduação em/ou depois de 2005 e quase 95\% realizavam seu primeiro treinamento, $55,1 \%$ cursavam o primeiro ano (R1) e $44,9 \%$, o segundo (R2). Cerca de $31 \%$ se concentravam nas especialidades "cirúrgicas" da medicina, 36\% nas especialidades "clínicas" da medicina e 32\% nas áreas de enfermagem, nutrição e saúde coletiva. A prevalência de TMC foi 39\% maior nos residentes médicos do que nos não médi$\cos (p=0,049)$. Os treinandos vinculados a especialidades "cirúrgicas" apresentaram prevalência de TMC 46\% maior do que a dos residentes de enfermagem, nutrição ou saúde coletiva ( $p=0,048$ ), mas não se observou diferença quando se comparou com os residentes das áreas "clínicas" ( $p$ > $0,05)$. Não se mostraram como fatores associados aos TMC o tempo de conclusão do curso de graduação, a existência de treinamento anterior e $o$ ano de treinamento $(p>0,05)$ (Tabela 2).

Tabela 1. Prevalência de TMC segundo variáveis sociodemográficas

\begin{tabular}{|c|c|c|c|c|c|c|}
\hline \multirow{2}{*}{ Variáveis } & \multicolumn{2}{|c|}{ Total $^{\mathrm{a}}$} & \multicolumn{2}{|c|}{ Prevalência } & \multirow{2}{*}{$\mathrm{RPb}^{\mathrm{b}}(\mathrm{IC}$ 95\%) } & \multirow{2}{*}{$\mathbf{p}$} \\
\hline & no & $\%$ & no & $\%$ & & \\
\hline Total & 178 & 100 & 91 & 51,1 & & \\
\hline \multicolumn{7}{|l|}{ Sexo } \\
\hline Feminino & 122 & 68,5 & 63 & 51,6 & $1,03(0,76-1,41)$ & $>0,05$ \\
\hline Masculino & 56 & 31,5 & 28 & 50,0 & 1,00 & \\
\hline \multicolumn{7}{|l|}{ Idade } \\
\hline$\leq 27$ & 111 & 62,4 & 58 & 52,3 & $1,06(0,79-1,43)$ & $>0,05$ \\
\hline$>27$ & 67 & 37,6 & 33 & 49,3 & 1,00 & \\
\hline \multicolumn{7}{|c|}{ Cidade onde residia anteriormente } \\
\hline Recife & 120 & 67,4 & 62 & 51,7 & $1,03(0,76-1,41)$ & $>0,05$ \\
\hline Outra cidade & 58 & 32,6 & 29 & 50,0 & 1,00 & \\
\hline \multicolumn{7}{|l|}{ Mora sozinho } \\
\hline Não & 157 & 88,2 & 81 & 51,6 & $1,08(0,67-1,74)$ & $>0,05$ \\
\hline Sim & 21 & 11,8 & 10 & 47,6 & 1,00 & $>0,05$ \\
\hline \multicolumn{7}{|l|}{ Estado conjugal } \\
\hline Solteiro & 131 & 73,6 & 67 & 51,1 & $1,00(0,72-1,39)$ & $>0,05$ \\
\hline Casado ou unido & 47 & 26,4 & 24 & 51,1 & 1,00 & \\
\hline \multicolumn{7}{|l|}{ Número de filhos } \\
\hline Nenhum & 161 & 90,4 & 86 & 53,4 & $1,82(0,86-3,85)$ & $>0,05$ \\
\hline 1 ou mais & 17 & 9,6 & 5 & 29,4 & 1,00 & \\
\hline
\end{tabular}

A soma do total em cada categoria varia em virtude da exclusão de indivíduos para os quais não havia informação

${ }^{\mathrm{b}} \mathrm{RP}$ : razão de prevalência. 
Tabela 2. Prevalência de TMC segundo formação profissional

\begin{tabular}{|c|c|c|c|c|c|c|}
\hline \multirow{2}{*}{ Variáveis } & \multicolumn{2}{|c|}{ Total $^{a}$} & \multicolumn{2}{|c|}{ Prevalência } & \multirow{2}{*}{$\operatorname{RPb}(I C 95 \%)$} & \multirow{2}{*}{$\mathrm{p}$} \\
\hline & no & $\%$ & no & $\%$ & & \\
\hline \multicolumn{7}{|l|}{ Médico } \\
\hline Sim & 121 & 68,0 & 68 & 56,2 & $1,39(0,98-1,98)$ & 0,049 \\
\hline Não & 57 & 32,0 & 23 & 40,4 & 1,00 & \\
\hline \multicolumn{7}{|l|}{ Ano de conclusão da graduação } \\
\hline 2005 e depois & 118 & 66,7 & 65 & 55,1 & $1,30(0,93-1,83)$ & $>0,05$ \\
\hline antes de 2005 & 59 & 33,3 & 25 & 42,4 & 1,00 & \\
\hline \multicolumn{7}{|l|}{ 1ậ residência } \\
\hline Sim & 169 & 94,9 & 88 & 52,1 & $1,56(0,61-3,98)$ & $>0,05$ \\
\hline Não & 9 & 5,1 & 3 & 33,3 & 1,00 & \\
\hline \multicolumn{7}{|l|}{ Ano do treinamento } \\
\hline Primeiro (R1) & 98 & 55,1 & 53 & 54,1 & $1,14(0,85-1,53)$ & $>0,05$ \\
\hline Segundo (R2) & 80 & 44,9 & 38 & 47,5 & 1,00 & \\
\hline \multicolumn{7}{|l|}{ Programa de residência } \\
\hline Medicina "Esp. Cirúrgicas" & 56 & 31,5 & 33 & 58,9 & $1,46(0,99-2,14)$ & 0,048 \\
\hline Medicina "Esp. Clínicas" & 65 & 36,5 & 35 & 53,8 & $1,33(0,91-1,97)$ & $>0,05$ \\
\hline Enfermagem ou Nutrição ou Saúde Coletiva & 57 & 32,0 & 23 & 40,4 & 1,00 & \\
\hline
\end{tabular}

${ }^{a}$ A soma do total em cada categoria varia em virtude da exclusão de indivíduos para os quais não havia informação.

${ }^{b} R P$ : razão de prevalência.

As três queixas, incluídas no SRQ-20, mais relatadas pelo conjunto dos residentes foram: "sentir-se nervoso, tenso ou preocupado" (73\%), "dormir mal" (69,1\%) e "cansar-se com facilidade" (61,2\%). Entre as mulheres, a queixa mais prevalente foi "sentir-se nervosa, tensa ou preocupada" (73,8\%) e entre os homens, "dormir mal" (76,8\%). De um total de 20 queixas, somente cinco delas apresentaram diferença estatisticamente significante entre os sexos, e em todos os casos a magnitude foi maior no sexo feminino. "Cansar-se com facilidade" e "ter sensações desagradáveis no estômago" foram, respectivamente, $62 \%(p<0,001)$ e $66 \%(p=0,02)$ mais relatadas pelas mulheres do que pelos homens $(p<0,02)$. No mesmo sentido, as prevalências das queixas "sentir dores de cabeça", "assustar-se com facilidade" e "chorar mais do que de costume" foram, respectivamente, duas $(<0,01)$, mais de três $(<0,001)$ e mais de seis $(<0,001)$ vezes maior nas mulheres do que nos homens (Tabela 3 ).

No grupo dos médicos, as três queixas mais frequentes foram: "sentir-se nervoso, tenso ou preocupado" (73,6\%), "dormir mal" (68,6\%) e "sentir-se cansado o tempo todo" (61,2\%). Já entre os profissionais não médicos, foram: "sentir-se nervoso, tenso ou preocupado" (71,9\%), "dormir mal" (70,2\%) e "cansar-se com facilidade" (68,4\%). Quando se compararam os dois grupos, a única queixa cuja prevalência mostrou diferença estatisticamente significante foi "dificuldade no serviço (seu trabalho Ihe causa sofrimento)", cerca de 2,7 vezes maior entre os médicos do que entre os não médicos $(p=0,001)$ (Tabela 4).

\section{DISCUSSÃO}

A prevalência global dos TMC entre os residentes foi de $51,1 \%$, magnitude considerada elevada quando comparada à encontrada em investigações envolvendo a população em geral (7\%-35\%) 1,3,2, ou grupos específicos, como estudantes da área de saúde (29,6\%-44,7\%) ${ }^{19-21}$ ou médicos, enfermeiros, auxiliares de enfermagem, agentes comunitários de saúde $(26 \%-43,3 \%)^{9,11,12,22,30,31}$

Nenhuma variável sociodemográfica se associou aos TMC, e isso já era esperado em relação à idade, uma vez que a amostra foi bastante homogênea. O diferencial por sexo é sempre observado em outros estudos que mostram maior prevalência no sexo feminino ${ }^{32-35}$. A explicação dada nesses casos é que a mulher se expõe à sobrecarga de papéis sociofamiliares, como a dupla jornada de trabalho, no lar e fora dele $^{32-35}$. Quando as perguntas do Self-Reporting Questionnaire (SRQ-20) foram avaliadas isoladamente, diferenças nas prevalências entre os sexos foram verificadas e em todas as situações a magnitude foi maior no sexo feminino. Coutinho et $a l^{33}$ alegam que a explicação para se detectar uma maior frequência de TMC no sexo feminino vincula-se a um possível viés de informação, gerado no momento da classificação. A adoção de pontos de corte diferenciados entre os sexos possivelmente minimizou a ocorrência desse bias neste estudo. 
Tabela 3. Prevalência das "queixas" do SRQ-20 segundo sexo

\begin{tabular}{|c|c|c|c|c|c|c|c|}
\hline \multirow{2}{*}{ Queixas } & \multicolumn{2}{|c|}{ Total } & \multicolumn{2}{|c|}{ Masculino } & \multicolumn{2}{|c|}{ Feminino } & \multirow{2}{*}{$\mathrm{p}$} \\
\hline & $n$ & $\%$ & $n$ & $\%$ & $n$ & $\%$ & \\
\hline Sente-se nervoso, tenso ou preocupado & 130 & 73,0 & 40 & 71,4 & 90 & 73,8 & $>0,05$ \\
\hline Dorme mal & 123 & 69,1 & 43 & 76,8 & 80 & 65,6 & $>0,05$ \\
\hline Cansa-se com facilidade & 109 & 61,2 & 24 & 42,9 & 85 & 69,7 & $<0,001$ \\
\hline Sente-se cansado o tempo todo & 108 & 60,7 & 31 & 55,4 & 77 & 63,1 & $>0,05$ \\
\hline Sente-se triste ultimamente & 102 & 57,3 & 27 & 48,2 & 75 & 61,5 & $>0,05$ \\
\hline Dificuldade para realizar atividades diárias & 99 & 55,6 & 26 & 46,4 & 73 & 59,8 & $>0,05$ \\
\hline Má digestão & 75 & 42,1 & 21 & 37,5 & 54 & 44,3 & $>0,05$ \\
\hline Tem sensações desagradáveis no estômago & 74 & 41,6 & 16 & 28,6 & 58 & 47,5 & 0,02 \\
\hline Dores de cabeça & 70 & 39,3 & 13 & 23,2 & 57 & 46,7 & $<0,01$ \\
\hline Dificuldade para tomar decisões & 66 & 37,1 & 18 & 32,1 & 48 & 39,3 & $>0,05$ \\
\hline Dificuldade de pensar com clareza & 62 & 34,8 & 19 & 33,9 & 43 & 35,2 & $>0,05$ \\
\hline Assusta-se com facilidade & 56 & 31,5 & 7 & 12,5 & 49 & 40,2 & $<0,001$ \\
\hline Dificuldade no serviço (seu trabalho Ihe causa sofrimento) & 54 & 30,3 & 18 & 32,1 & 36 & 29,5 & $>0,05$ \\
\hline Chora mais do que de costume & 46 & 25,8 & 3 & 5,4 & 43 & 35,2 & $<0,001$ \\
\hline Perda do interesse pelas coisas & 42 & 23,6 & 11 & 19,6 & 31 & 25,4 & $>0,05$ \\
\hline Falta de apetite & 25 & 14,0 & 11 & 19,6 & 14 & 11,5 & $>0,05$ \\
\hline Tremores nas mãos & 23 & 12,9 & 7 & 12,5 & 16 & 13,1 & $>0,05$ \\
\hline Incapacidade de desempenhar um papel útil na vida & 16 & 9,0 & 5 & 8,9 & 11 & 9,0 & $>0,05$ \\
\hline Sente-se uma pessoa inútil, sem préstimo & 12 & 6,7 & 4 & 7,1 & 8 & 6,6 & $>0,05$ \\
\hline Tem tido ideia de acabar com a vida & 5 & 2,8 & 2 & 3,6 & 3 & 2,5 & $>0,05$ \\
\hline
\end{tabular}

Tabela 4. Prevalência das "queixas" do SRQ-20 segundo profissão

\begin{tabular}{|c|c|c|c|c|c|}
\hline \multirow{2}{*}{ Queixas } & \multicolumn{2}{|c|}{ Médico } & \multicolumn{2}{|c|}{ Não médico } & \multirow{2}{*}{$p$} \\
\hline & $\mathrm{n}$ & $\%$ & $\mathrm{n}$ & $\%$ & \\
\hline Sente-se nervoso, tenso ou preocupado & 89 & 73,6 & 41 & 71,9 & $>0,05$ \\
\hline Dorme mal & 83 & 68,6 & 40 & 70,2 & $>0,05$ \\
\hline Sente-se cansado o tempo todo & 74 & 61,2 & 34 & 59,6 & $>0,05$ \\
\hline Dificuldade para realizar atividades diárias & 71 & 58,7 & 28 & 49,1 & $>0,05$ \\
\hline Sente-se triste ultimamente & 70 & 57,9 & 32 & 56,1 & $>0,05$ \\
\hline Cansa-se com facilidade & 70 & 57,9 & 36 & 68,4 & $>0,05$ \\
\hline Má digestão & 54 & 44,6 & 21 & 36,8 & $>0,05$ \\
\hline Tem sensações desagradáveis no estômago & 50 & 41,3 & 24 & 42,1 & $>0,05$ \\
\hline Dificuldade de pensar com clareza & 47 & 38,8 & 15 & 26,3 & $>0,05$ \\
\hline Dores de cabeça & 46 & 38,0 & 24 & 42,1 & $>0,05$ \\
\hline Dificuldade para tomar decisões & 46 & 38,0 & 20 & 35,1 & $>0,05$ \\
\hline Dificuldade no serviço (seu trabalho Ihe causa sofrimento) & 46 & 38,0 & 8 & 14,0 & 0,001 \\
\hline Assusta-se com facilidade & 34 & 28,1 & 22 & 38,6 & $>0,05$ \\
\hline Perda do interesse pelas coisas & 28 & 23,1 & 14 & 24,6 & $>0,05$ \\
\hline Chora mais do que de costume & 26 & 21,5 & 20 & 35,1 & $=0,05$ \\
\hline Falta de apetite & 18 & 14,9 & 7 & 12,3 & $>0,05$ \\
\hline Tremores nas mãos & 18 & 14,9 & 5 & 8,8 & $>0,05$ \\
\hline Incapacidade de desempenhar um papel útil na vida & 11 & 9,1 & 5 & 8,8 & $>0,05$ \\
\hline Sente-se uma pessoa inútil, sem préstimo & 9 & 7,4 & 3 & 5,3 & $>0,05$ \\
\hline Tem tido ideia de acabar com a vida & 4 & 3,3 & 1 & 1,8 & $>0,05$ \\
\hline
\end{tabular}


A migração entre cidades não esteve associada aos TMC no estudo de Almeida-Filho et al. ${ }^{36}$, e a condição de morar sozinho foi considerada fator de risco por Coutinho et al. ${ }^{33}$ e Lima ${ }^{37}$.

Em relação à vida conjugal, a ausência na amostra de situações que aumentam o risco de TMC, como divórcio, separação e filhos com idade até $14^{33,35}$, possivelmente explicaria a igualdade estatística nas prevalências observadas.

A prevalência de TMC em residentes médicos foi 39\% maior do que em residentes não médicos $(p=0,049)$. Caso o tamanho da amostra fosse maior, possivelmente a precisão dessa associação aumentaria. Hipóteses vinculadas à organização do processo do trabalho médico podem explicar essa diferença ${ }^{23}$. O perfil sobre o relato das "queixas" foi muito semelhante nos dois grupos. Somente uma "queixa" apresentou diferença estatisticamente significante: os médicos vincularam seu trabalho com sofrimento.

Ao comparar a saúde mental de médicos com a de outros profissionais, alguns autores verificaram naqueles maior incidência de suicídio e de reações depressivas ${ }^{38-40}$. A prevalência de TMC observada nos residentes médicos $(56,2 \%)$ foi mais elevada do que a encontrada em professores (entre $44 \%$ e $50,3 \%)^{6,41}$ e em médicos-residentes americanos $(50 \%)^{42}$.

O treinamento vinculado ao trabalho em ambiente cirúrgico, por ser mais estressante do que aquele realizado nas áreas de enfermagem, nutrição e saúde coletiva, constitui uma possibilidade de explicação para o fato de se observar uma prevalência de TMC maior nos residentes médicos das especialidades cirúrgicas que requerem habilidade técnica, controle emocional, autoconfiança, maior pragmatismo, capacidade para direcionar ações para resolução de problemas num curto espaço de tempo ${ }^{14}$.

As diferenças de prevalência de TMC encontradas entre os profissionais médicos e das outras áreas de saúde pesquisadas pode ser em parte explicada pelas particularidades entre elas. Muito embora todas as profissões investigadas lidem com questões de saúde, o residente médico enfrenta situações de maior responsabilidade diante do paciente. Na literatura são relatados fatores estressantes como a relação médico-paciente, o comunicar doenças graves, o lidar com a morte, o medo de adquirir doenças contagiosas, rodízios em ambientes diferentes, isolamento familiar e social, fadiga, privação de sono, sobrecarga de trabalho, pavor de cometer erros, entre outras demandas que envolvem a formação médica ${ }^{13-18,43,44}$.

O tempo de conclusão do curso e o fato de o residente estar no primeiro treinamento não se mostraram associados aos TMC. Alguns estudos ${ }^{14,15}$ mostram que a prevalência de depressão e ansiedade é maior no primeiro ano de treinamento, com tendência à queda até sua conclusão.

Algumas limitações vinculadas ao desenho de estudo poderiam relativizar essas conclusões. Trata-se de um corte transversal que avalia em um só momento a "exposição" e o "efeito", limitando ou impedindo o estabelecimento da sequência temporal correta entre eles. Não se pode afastar a possibilidade de que uma parcela dos residentes já sofresse de TMC antes de entrar nos programas de residência, o que diminuiria a força de associação entre a vinculação ao treinamento e a ocorrência do efeito. Uma investigação do tipo coorte ajudaria a esclarecer as dúvidas quanto à magnitude e aos fatores de risco.

A estratégia montada para prevenir perdas se mostrou eficaz, reduzindo o viés de seleção. O uso de questionário autoaplicável e o anonimato das respostas minimizaram o viés de informação concernente a perguntas sobre a intimidade dos pesquisados. O elevado grau de preenchimento médio das questões não comprometeu a análise dos dados.

O ponto de corte diferenciado entre os sexos para o SRQ20 procurou evitar bias de classificação 6,19,21,24,25. Quanto ao risco de subestimação do evento não parece ter ocorrido, uma vez que foi observada uma elevada prevalência de TMC na amostra. Deve-se ressaltar, ainda, que o instrumento do SRQ-20 permite apenas a suspeição de um caso de transtorno mental, sendo a entrevista psiquiátrica o padrão-ouro para o diagnóstico.

\section{CONCLUSÃO}

O estudo revelou elevada magnitude de TMC na população de treinandos em programas de residência no estado de Pernambuco, além de mostrar diferenciais nas prevalências do problema entre médicos e não médicos e no perfil de "queixas" do SRQ-20 entre os sexos.

Esses resultados servem de reflexão para educadores, preceptores, gestores e coordenadores dos programas e serviços de saúde no sentido de elaborar estratégias para o enfrentamento da questão que afeta a qualidade de vida desses profissionais em formação.

\section{CONTRIBUIÇÕES INDIVIDUAIS}

Carla Novaes Carvalho - Participou da concepção do projeto, revisão da literatura, coleta e análise dos dados, interpretação dos resultados, redação do artigo, aprovação da versão final a ser publicada.

Djalma Agripino de Melo-Filho - Contribuiu com a concepção do projeto, orientação sobre a literatura, análise e interpretação dos dados, redação do artigo e revisão crítica relevante do conteúdo e aprovação final da versão a ser publicada.

João Alberto Gomes de Carvalho - Colaborou na concepção do projeto, revisão do artigo e aprovação da versão final.

Ana Carla Guedes de Amorim - Participou na concepção e coleta de dados do projeto, da redação do artigo e da aprovação da versão a ser publicada. 


\section{FONTES DE FINANCIAMENTO}

Nenhuma

\section{AGRADECIMENTOS}

Agradecimento especial a Igor Henrique Figueira da Silva, pelo grande auxílio prestado na laboriosa coleta de dados.

\section{CONFLITO DE INTERESSES}

Os autores declaram que não houve conflito de interesses.

\section{REFERÊNCIAS}

1. Goldberg D, Huxley P. Common mental disorders: a bio-social model. 1st ed. London: Tavistock/Routledge; 1992. 194p.

2. Mari JJ, Jorge MR. Transtornos psiquiátricos na clínica geral. Psychiatry On-line Brazil. 1997;2. Disponível em: <http://www.polbr.med.br/ano97/tpqcm.php>. Acesso em: 22 jul. 2011.

3. Lopez AD, Mathers CD, Ezzati M, Jamison DT, Murray CJL, editors; WHO. Global burden of disease and risk factors. New York: Oxford University Press; 2006.

4. Ludermir AB, Melo-Filho DA. Condições de vida e estrutura ocupacional associadas a transtornos mentais comuns. Rev Saude Publica. 2002;36(2):213-21.

5. Lima MS, Beria JU, Tomasi E, Conceição AT, Mari JJ. Stressful life events and minor psychiatric disorders: an estimate of the population attributable fraction in a Brazilian communitybased study. Int J Psychiatry Med. 1996;26(2):211-22.

6. Porto LA, Carvalho FM, Oliveira NF, Silvany Neto AM, Araújo TM, Reis EJFB, et al. Associação entre distúrbios psíquicos e aspectos psicossociais do trabalho de professores. Rev Saude Publica. 2006;40(5):818-26.

7. Souza SF, Carvalho FM, Araújo TM, Porto LA. Fatores psicossociais do trabalho e transtornos mentais comuns em eletricitários. Rev Saude Publica. 2010;44(4):710-7.

8. Ulhoa MA, Marqueze EC, Lemos LC, Silva LG, Silva AA, Nehme P, et al. Distúrbios psíquicos menores e condições de trabalho em motoristas de caminhão. Rev Saude Publica. 2010:44(6):1130-6.

9. Pitta A. Hospital: dor e morte como ofício. São Paulo: HUCITEC; 1994. 198p.

10. Nascimento Sobrinho CL, Carvalho FM, Bonfim TA, Cirino CA, Ferreira IS. Condições de trabalho e saúde mental dos médicos de Salvador, Bahia, Brasil. Cad Saude Publica. 2006:22(1):131-40

11. Cabana MC, Ludermir AB, Silva ER, Ferreira ML, Pinto ME. Transtornos mentais comuns em médicos e seu cotidiano de trabalho. J Bras Psiquiatr. 2007;56(1):33-40.

12. Silva AT, Menezes PR. Esgotamento profissional e transtornos mentais comuns em agentes comunitários de saúde. Rev Saude Publica. 2008:42(5):921-9.

13. Smith JW, Denny WF, Witzke DB. Emotional impairment in internal medicine house staff. JAMA. 1986;255(9):1155-8.

14. Nogueira-Martins LA. Residência médica: estresse e crescimento. 1a ed. São Paulo: Casa do Psicólogo; 2005. 201p.

15. Campbell J, Prochazka AV, Yamashita T, Gopal R. Predictors of persistent burnout in internal medicine residents: a prospective cohort study. Acad Med. 2010;85(10):1630-4.

16. Resident Services Committee, Association of Program Directors in Internal Medicine. Stress and impairment during residency training: strategies for reduction, identification, and management. Ann Intern Med. 1988;109(2):154-61.

17. Girard DE, Hickam DH, Gordon GH, Robison RO. A prospective study of internal medicine residents' emotions and attitudes throughout their training. Acad Med. 1991;66(2):111-4.

18. Small GW. House officer stress syndrome. Psychosomatics. 1981;22(10):860-9

19. Almeida AM, Godinho TM, Bitencourt AG, Teles MS, Silva AS, Fonseca DC, et al. Common mental disorders among medical students. J Bras Psiquiatr. 2007;56(4):245-51.
20. Lima MC, Domingues MS, Cerqueira AT. Prevalência e fatores de risco para transtornos mentais comuns entre estudantes de medicina. Rev Saude Publica. 2006;40(6):1035-41.

21. Facundes VL, Ludermir AB. Common mental disorders among health care students. Rev Bras Psiquiatr. 2005;27(3):194-200.

22. Araújo TM, Aquino E, Menezes G, Santos CO, Aguiar L. Aspectos psicossociais do trabalho e distúrbios psíquicos entre trabalhadoras de enfermagem. Rev Saude Publica. 2003;37(4):424-33.

23. Carvalho CN. Transtornos mentais comuns associados ao processo de trabalho e qualidade de vida em pós-graduandos dos programas de residência na área de saúde no Recife [dissertação]. [Recife (PE)]: Universidade Federal de Pernambuco; 2008. 118p.

24. Harding TW, De Arango MV, Baltazar J, Climent CE, Ibrahim HH, Ladrido-Ignacio L, et al. Mental disorders in primary health care: a study of their frequency and diagnosis in four developing countries. Psychol Med. 1980;10(2):231-41.

25. WHO. A user's guide to the Self-Reporting Questionnaire. Geneva: World Health Organization; 1994. 81p.

26. Santos KO, Araújo TM, Oliveira NF. Estrutura fatorial e consistência interna do Self-Reporting Questionnaire (SRQ-20) em população urbana. Cad Saude Publica. 2009;25(1):214-22.

27. Iacoponi E, Mari JJ. Reliability and factor structure of the Portuguese version of Self-Reporting Questionnaire. Int J Soc Psychiatry. 1989;35(3):213-22.

28. Maragno L, Goldbaum M, Gianini RJ, Novaes HM, Cesar CL. Prevalência de transtornos mentais comuns em populações atendidas pelo Programa Saúde da Família (QUALIS) no Município de São Paulo, Brasil. Cad Saude Publica. 2006;22(8):1639-48.

29. Rocha SV, Almeida MM, Araújo TM, Virtuoso-Júnior JS. Prevalência de transtornos mentais comuns entre residentes em áreas urbanas de Feira de Santana, Bahia. Rev Bras Epidemiol. 2010;13(4):630-40

30. Nascimento Sobrinho CL, Carvalho FM, Bonfim TA, Cirino CA, Ferreira IS. Condiçōes de trabalho e saúde mental dos médicos de Salvador, Bahia, Brasil. Cad Saude Publica. 2006;22(1):131-40

31. Braga $L C$, Carvalho LR, Binder MC. Condições de trabalho e transtornos mentais comuns em trabalhadores da rede básica de saúde de Botucatu (SP). Ciênc Saúde Coletiva. 2010;15(Supl 1):1585-96.

32. Cesar CL, Carandina L, Alves MC, Barros MB, Goldbaum M. Saúde e condição de vida em São Paulo. Inquérito multicêntrico de saúde no estado de São Paulo - ISA/SP. São Paulo: USP/FSP; 2005. 212p

33. Coutinho ES, Almeida-Filho N, Mari JJ. Fatores de risco para morbidade psiquiátrica menor: resultados de um estudo transversal em três áreas urbanas no Brasil. Rev Psiq Clín. 1999;26(5):246-56

34. Justo LP, Calil HM. Depressão: o mesmo acometimento para homens e mulheres? Rev Psiq Clín. 2006;33(2):74-9.

35. Araújo TM, Pinho PD, Almeida MM. Prevalência de transtornos mentais comuns em muIheres e sua relação com as características sociodemográficas e 0 trabalho doméstico. Rev Bras Saúde Matern Infant. 2005;5(3):337-48

36. Almeida-Filho N, Mari JJ, Coutinho ESF. Migração, inserção produtiva e saúde mental na modernidade tardia: novas evidências do estudo multicêntrico de morbidade psiquiátrica em áreas metropolitanas brasileiras. Rev Psiq Clín. 1999;26(5):237-45.

37. Lima MS. Epidemiologia e impacto social. Rev Bras Psiquiatr. 1999;21(Supl 1):1-5.

38. Machado MH. Os médicos no Brasil: um retrato da realidade. Rio de Janeiro: FIOCRUZ; 1997. $244 p$.

39. Meleiro AM. Suicídio entre médicos e estudantes de medicina. Rev Ass Med Bras. $1998: 44(2): 135-40$

40. Tyssen R. Health problems and the use of health services among physicians: a review article with particular emphasis on Norwegian studies. Ind Health. 2007;45(5):599-610.

41. Gasparini SM, Barreto SM, Assunção AA. Prevalência de transtornos mentais comuns em professores da rede municipal de Belo Horizonte, Minas Gerais, Brasil. Cad Saude Publica. 2006:22(12):2679-91.

42. Firth-Cozens J. Emotional distress in junior house officers. Br Med J (Clin Res Ed). 1987;295(6597):533-6.

43. Hsu K, Marshall V. Prevalence of depression and distress in a large sample of Canadian residents, interns, and fellows. Am J Psychiatry. 1987;144(12):1561-6.

44. Ripp J, Fallar R, Babyatsky M, David R, Reich L, Korenstein D. Prevalence of resident burnout at the start of training. Teach Learn Med. 2010;22(3):172-5. 